\title{
Large-cell neuroendocrine carcinoma arising in the endometrium: A case report
}

\author{
JUMPEI OGURA $^{1,2}$, YASUSHI ADACHI ${ }^{3}$, KOJI YASUMOTO ${ }^{1,2}$, AKIHARU OKAMURA $^{4}$, HIROFUMI NONOGAKI ${ }^{1}$, \\ KAZUYO KAKUI $^{1,2}$, KOJI YAMANOI ${ }^{1,2}$, KOH SUGINAMI $^{1,2}$, TAKASHI KOYAMA ${ }^{5}$ and SUSUMU IKEHARA ${ }^{6}$ \\ ${ }^{1}$ Department of Obstetrics and Gynecology, Toyooka Hospital, Toyooka, Hyogo 668-8501; ${ }^{2}$ Department of Obstetrics \\ and Gynecology, Kyoto University, Kyoto, Kyoto 606-8501; ${ }^{3}$ Department of Diagnostic Pathology, Toyooka Hospital, \\ Toyooka, Hyogo 668-8501; ${ }^{4}$ Department of Diagnostic Pathology, Kakogawa Central City Hospital, Kakogawa, \\ Hyogo 675-8611; ${ }^{5}$ Department of Radiology, Toyooka Hospital, Toyooka, Hyogo 668-8501; \\ ${ }^{6}$ Professor Emeritus, Kansai Medical University, Hirakata, Osaka 573-1010, Japan
}

Received September 1, 2017; Accepted January 17, 2018

DOI: $10.3892 / \mathrm{mco} .2018 .1583$

\begin{abstract}
Large-cell neuroendocrine carcinoma (LCNEC) of the endometrium is an extremely rare, high-grade malignant tumor. We herein report a case of a rapidly growing LCNEC arising in the endometrium. A 52-year-old woman was referred to Toyooka Hospital (Tooyoka, Japan) due to genital bleeding in February 2016. There had been no abnormalities on a regular gynecological and physical examination 3 months prior to the consultation. Imaging (computed tomography and magnetic resonance imaging) and a pelvic examination revealed a tumor sized $16.9 \times 8.4 \times 7.8 \mathrm{~mm}$ occupying the intrauterine cavity and extending into the vaginal cavity. Multiple metastatic pelvic and paraaortic lymph nodes were also identified. Continuous bleeding from the tumor was observed, and a blood examination revealed anemia, which was likely due to that bleeding. Biopsy of the tumor was performed, and large atypical cells were identified. The tumor cells were negative for cytokeratin AE1/AE3 and chromogranin A, but positive for CD56 and synaptophysin. There was also an abundance of Ki-67-positive cells in the tumor, altogether suggesting that the tumor was an LCNEC. The patient succumbed to the disease 36 days after the first consultation. Based on the findings of the present case and previously published cases, LCNECs arising in the endometrium may progress rapidly and are associated with an unfavourable outcome. LCNEC should be included in the differential diagnosis in cases of rapidly growing tumors of the uterine corpus.
\end{abstract}

Correspondence to: Dr Yasushi Adachi, Department of Diagnostic Pathology, Toyooka Hospital, 1094 Tobera, Toyooka, Hyogo 668-8501, Japan

E-mail: adachiya250@gmail.com

Key words: large-cell neuroendocrine carcinoma, uterus, endometrium, poor prognosis, negative for cytokeratin

\section{Introduction}

Large-cell neuroendocrine carcinoma (LCNEC) was first reported in the lung (1). LCNECs have since been reported in several locations, including the mediastinum, head and neck, pancreas, gallbladder, intestine and gynecological organs (2,3). Among gynecological organs, LCNECs more frequently occur in the uterine cervix rather than the uterine corpus. To the best of our knowledge, after the first such case was reported in 2004 (4), only 18 cases of LCNEC arising in the uterine corpus have been published to date (5-8). Due to the small number of reported cases, LCNECs may be difficult to diagnose. We herein present a case of LCNEC arising in the endometrium, with aim to describe the histological characteristics of this tumor and emphasize its rapid progression and poor prognosis.

\section{Case report}

A 52-year-old woman (gravida 6, para 4), with no history of gynecological disorders, was referred to Toyooka Hospital (Toyooka, Japan) due to genital bleeding in February 2016. The patient had been taking a low-dose contraceptive pill for 18 months prior to the consultation to control functional bleeding. Regular gynecological physical examinations 3 months prior to the consultation failed to identify any abnormalities. The patient consulted her primary care doctor due to continuous genital bleeding for 50 days. The doctor diagnosed the patient with anemia [hemoglobin $(\mathrm{Hb})$ level, $8.8 \mathrm{~g} / \mathrm{dl}$ ] and detected a uterine cervical polyp; the patient was subsequently referred to Toyooka Hospital. Upon pelvic examination at the Department of Gynecology, a cauliflowershaped tumor, $\sim 80 \mathrm{~mm}$ in diameter, was identified in the vaginal cavity. Continuous bleeding from the tumor surface was observed. Anemia was also observed on blood examination. The tumor appeared to develop from the uterine os, suggesting that it originated in the uterine cavity and extended into the vaginal cavity. Examination using ultrasound revealed a tumor occupying the entire uterine cavity and was attached to the fundus through a stalk. Magnetic resonance 

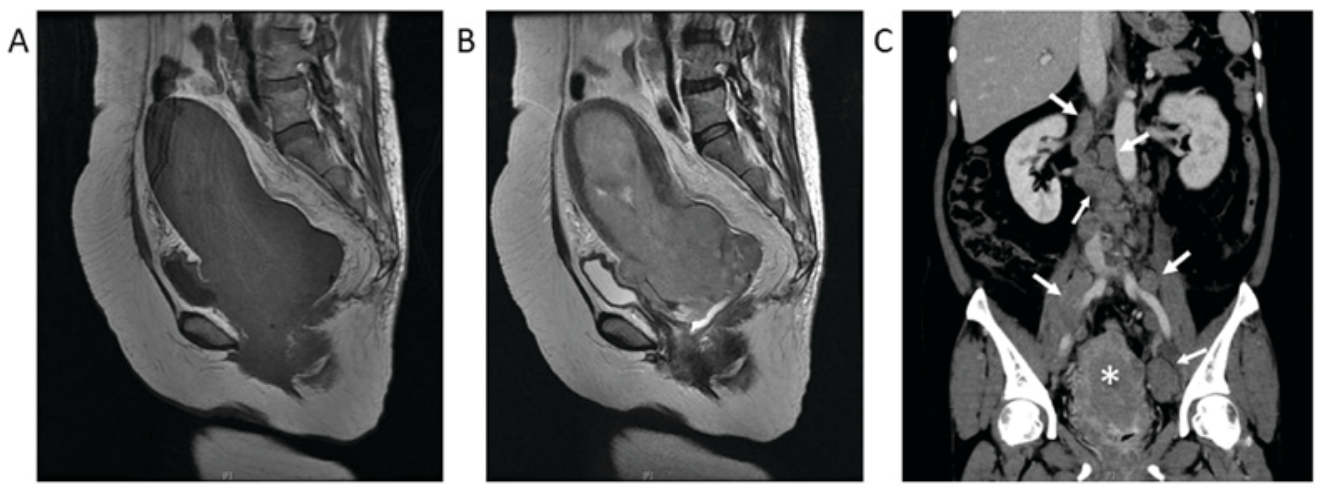

Figure. 1 Diagnostic imaging of the tumor. (A) Sagittal T1-weighted magnetic resonance imaging (MRI) without fat saturation and (B) T2-weighted MRI of the abdomen and pelvis revealed a sizeable homogeneous tumor in the endometrial cavity, which protruded into the vaginal cavity. (C) Coronal contrast-enhanced computed tomography scan revealed a heterogeneously enhanced uterine tumor $\left(^{*}\right)$ with enlargement of multiple para aortic and external iliac lymph nodes, and partially necrotic internal iliac lymph nodes (arrows).
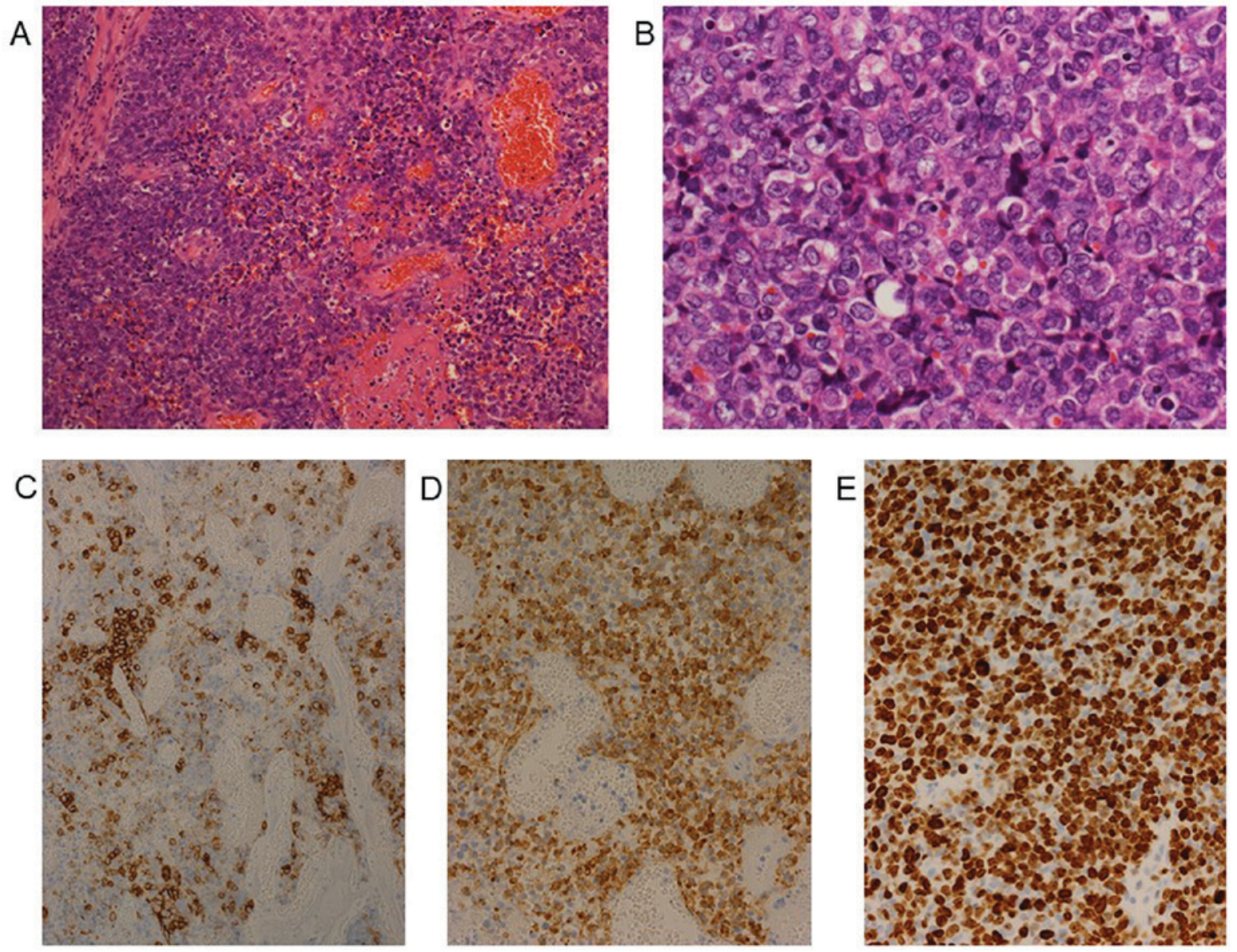

Figure. 2 Histological findings from tumor biopsy. The biopsy sample was composed of medium-large atypical tumor cells with prominent nucleoli, and of necrotic tissue: Original magnification of objective lens, (A) x20 and (B) x60. On immunohistochemical staining, the tumor cells were positive for (C) CD56 and (D) synaptophysin; (E) the Ki-67 index was $\sim 85 \%$ (original magnification of objective lens, $\mathrm{x} 40$ ).

imaging examination revealed a homogeneous tumor, sized $16.9 \times 8.4 \times 7.8 \mathrm{~mm}$, in the endometrial cavity, protruding into the vaginal cavity. A coronal contrast-enhanced computed tomography scan revealed a heterogeneously enhanced uterine tumor and enlargement of the paraaortic lymph nodes, external iliac lymph nodes, and partially necrotic internal iliac lymph nodes, suggesting lymph node metastasis. There was no detectable invasion of the tumor into the bladder or rectum on diagnostic imaging (Fig. 1). Laboratory data on admission included $\mathrm{Hb} 8.7 \mathrm{~g} / \mathrm{dl}$, C-reactive protein $8.73 \mathrm{mg} /$ $\mathrm{dl}$, creatinine $0.77 \mathrm{mg} / \mathrm{dl}$, lactate dehydrogenase $814 \mathrm{U}$ and carbohydrate antigen-125 36.5 U/ml. Histological examination of a biopsy sample from the tumor revealed atypical cells and necrotic tissue. The tumor cells were medium-large in size, with abundant cytoplasm and prominent nucleoli (Figs. 2A and B). Immunohistochemically, the tumor cells 
were positive for CD56 and synaptophysin (Figs. 2C and D), and negative for cytokeratin AE1/AE3, chromogranin A, P40, $\alpha$-smooth muscle actin (SMA), S100, CD10 and CD34. The Ki-67 index was $\sim 85 \%$ (Fig. 2E). Based on the histological characteristics and results of the immunochemical staining, the tumor was diagnosed as LCNEC. The blood levels of neuron-specific enolase (NSE) and proGRP was measured, and revealed that the level of proGRP was normal, but the level of NSE was extremely high $(240.4 \mathrm{ng} / \mathrm{ml}$; normal range, $\leq 10 \mathrm{ng} / \mathrm{ml}$ ). Due to the poor condition of the patient and extent of tumor spread, palliative care using morphine hydrochloride $(600 \mu \mathrm{g} / \mathrm{kg} / \mathrm{day})$ was administered in an attempt to alleviate the symptoms. However, the tumor rapidly progressed and the patient succumbed to the disease 36 days after admission.

\section{Discussion}

Erhan et al first reported LCNEC arising in the endometrium in 2004 (4). To the best of our knowledge, only 18 cases (excluding the present case) have been reported in the literature to date. In 2013, Nguyen et al summarized 13 cases of endometrial LCNEC (5), and Kobayashi et al (6) reviewed 16 cases in 2017. While reviewing the relevant literature, 2 additional reports were identified $(7,8)$. Thus, including the case presented herein, there are a total of 19 reported cases of endometrial LCNEC in patients aged 40-88 years [mean, 62 years; standard deviation (SD), 14.8 years].

Cases of LCNEC arising in the cervix have also been reported. Gilks et al reported 12 cases of LCNEC arising in the uterine cervix (cervical LCNEC) in patients aged 21-62 years (mean, 34 years) (9). Wang et al reported a mean age of 42 years and a SD of 11.3 years across 4 cases (10). Therefore, the mean age of patients with endometrial LCNEC appears to be higher compared with that of patients with cervical LCNEC.

The mean age at diagnosis of endometrioid carcinoma of the endometrium (the most common type of endometrial carcinoma) is $\sim 63$ years, while the majority of patients with microinvasive squamous cell carcinoma ( $\mathrm{SqCC}$ ) arising in the uterine cervix are aged 35-46 years (11). Microinvasive SqCC of the uterine cervix represents the early stage of $\mathrm{SqCC}$, which is the most common type of carcinoma in the uterine cervix. These results suggest that the susceptible age range for LCNEC is similar to that of endometrioid carcinoma of the endometrium and $\mathrm{SqCC}$ of the uterine cervix. Of the 19 cases of endometrial LCNEC, 2 cases, including our patient, were diagnosed by biopsy only. Therefore, it remains unclear whether the tumors were composed purely of LCNEC cells, or incorporated other histological type(s). Of the 17 resected tumors, 6 contained an adenocarcinoma component, 5 contained endometrioid carcinoma and 1 contained serous carcinoma $(8,12,13)$. These results suggest that adenocarcinoma of the endometrium may transform into LCNEC in a proportion of the cases. Even if the histological diagnosis is pure LCNEC, this may be the result of LCNEC cells overwhelming any other type of carcinoma and dominating the entire tumor. As LCNEC has a high Ki-67 index and exhibits rapid growth, the prognosis of endometrial LCNEC is poor. Of the 19 patients, 8 (42\%) succumbed to the disease during the follow-up period. The mean period of follow-up of the 8 patients was 7 months (SD, 7.7 months). The survival rate was $46 \%$ at 1 year and $11 \%$ at 5 years after the diagnosis of LCNEC. In the present case, the patient succumbed to rapid tumor growth $\sim 1$ month after the hospital consultation. Similar rapidly progressing cases of endometrial LCNEC have been reported by Nguyen et al (5) and Makihara et al (14).

For diagnosis of LCNEC, histological examination is necessary. However, it has been reported that neuroendocrine carcinomas, including small-cell carcinoma and LCNEC, do not always express cytokeratin AE1/AE3, which is commonly used as an epithelial marker (15). In the present case, the tumor was negative for cytokeratin AE1/AE3. Therefore, sarcoma was first suspected and staining with anti-a-SMA, anti-S100 and anti-CD10 antibodies was performed; the tumor was negative for all three. Thereafter, staining for neuroendocrine markers was performed and the blood levels of NSE and proGRP were measured, which confirmed the diagnosis of LCNEC. If endometrial LCNEC had been considered earlier in the differential diagnosis, the patient may have been diagnosed sooner. Therefore, when a rapidly growing tumor is detected, even if that tumor is negative for cytokeratin, the possibility of LCNEC should be taken into consideration.

In conclusion, we herein present a rare case of LCNEC with rapid growth and poor prognosis. When the tumor is cytokeratin-negative, it must be carefully examined before a definitive diagnosis is reached. Other cytokeratin-negative carcinomas in addition to neuroendocrine tumors, such as sarcoma and hematopoietic tumors, should also be considered.

\section{Acknowledgements}

The authors would like to thank Ms. H. Ogaki, Mr. K Nagaoka, Mr. T. Kuge and Mr. H. Takenaka of the Toyooka Hospital for their expert technical assistance.

\section{Funding}

No funding was received.

\section{Availability of data and materials}

The datasets used and/or analyzed during the current study are available from the corresponding author on reasonable request.

\section{Authors' contributions}

JO, YA, KYas, AO, HN, KK, KYam, KS, TK and SI designed the study. JO, KYas, HN, KK, KYam, KS, TK and YA analyzed and interpreted the patient data. JO and YA were major contributors in writing the manuscript. AO, SI and YA performed the histological examination of the tumor and performed histological diagnosis.

\section{Ethics approval and consent to participate}

The Ethics Committee of the Toyooka Hospital (Toyooka, Japan) approved the present study and the patient provided written informed consent. 


\section{Consent for publication}

The patient approved the publication of their data within this case report.

\section{Competing interests}

The authors confirm that they have no competing interests.

\section{References}

1. Travis WD, Linnoila RI, Tsokos MG, et al: Neuroendocrine tumors of the lung with proposed criteria for large-cell neuroendocrine carcinoma. An ultrastructural, immunohistochemical and flow cytometric study of 35 cases. Am J Surg Pathol 15 $529-553,1991$.

2. Lukina O, Gorbunkov S, Dvorakovskaja I, Varlamov V and Akopov A: Fast-growing large cell neuroendocrine carcinoma of mediastinum. Ann Thorac Surg 91: 1618-1620, 2011.

3. Faggiano A, Sabourin JC, Ducreux M, et al: Pulmonary and extrapulmonary poorly differentiated large cell neuroendocrine carcinomas: diagnostic and prognostic features. Cancer 110 265-274, 2007.

4. Erhan Y, Dikmen Y, Yucebilgin MS, Zekioglu O, Mgoyi L and Terek MC: Large cell neuroendocrine carcinoma of the uterine corpus metastatic to brain and lung: case report and review of the literature. Eur J Gynaecol Oncol 25: 109-112, 2004.

5. Nguyen ML, Han L, Minors AM, et al: Rare large cell neuroendocrine tumor of the endometrium: A case report and review of the literature. Int J Surg Case Rep 4: 651-655, 2013.

6. Kobayashi A, Yahata T, Nanjo S, et al: Rapidly progressing largecell neuroendocrine carcinoma arising from the uterine corpus: A case report and review of the literature. Mol Clin Oncol 6: 881-885, 2017.
7. Froio E, D' Adda T, Fellegara G, et al: Uterine carcinosarcoma metastatic to the lung as large-cell neuroendocrine carcinoma with synchronous sarcoid granulomatosis. Lung Cancer 64: 371-377, 2009.

8. Ono K, Yokota NR, Yoshioka E, et al: Metastatic large cell neuroendocrine carcinoma of the lung arising from the uterus: A pitfall in lung cancer diagnosis. Pathol Res Pract 212: 654-657, 2016.

9. Gilks CB, Young RH, Gersell DJ and Clement PB: Large cell neuroendocrine [corrected] carcinoma of the uterine cervix: a clinicopathologic study of 12 cases. Am J Surg Pathol 21: 905-914, 1997.

10. Wang KL, Yang YC, Wang TY, et al: Neuroendocrine carcinoma of the uterine cervix: A clinicopathologic retrospective study of 31 cases with prognostic implications. J Chemother 18: 209-216, 2006.

11. Kurman RJ, Ronnett, B.M., Sherman, M.E., Wilkinson, E.J.: Tumor of the cervix. ARP press, Silver Spring, Maryland, 2010.

12. Mulvany NJ and Allen DG: Combined large cell neuroendocrine and endometrioid carcinoma of the endometrium. Int J Gynecol Pathol 27: 49-57, 2008.

13. Posligua L, Malpica A, Liu J, Brown J and Deavers MT: Combined large cell neuroendocrine carcinoma and papillary serous carcinoma of the endometrium with pagetoid spread. Arch Pathol Lab Med 132: 1821-1824, 2008.

14. Makihara N, Maeda T, Nishimura M, et al: Large cell neuroendocrine carcinoma originating from the uterine endometrium: a report on magnetic resonance features of 2 cases with very rare and aggressive tumor. Rare Tumors 4: e37, 2012.

15. McCluggage WG, Oliva E, Connolly LE, McBride HA and Young RH: An immunohistochemical analysis of ovarian small cell carcinoma of hypercalcemic type. Int J Gynecol Pathol 23: 330-336, 2004 